\title{
Effect of the solar activity variation on the Global Ionosphere Thermosphere Model (GITM)
}

\author{
Davide Masutti $^{1}$, Günther March ${ }^{2}$, Aaron J. Ridley ${ }^{3}$, and Jan Thoemel ${ }^{1}$ \\ ${ }^{1}$ Von Karman Institute for Fluid Dynamics, Sint-Genesius-Rode 1640, Belgium \\ ${ }^{2}$ Delft University of Technology, Delft 2629 HS, the Netherlands \\ ${ }^{3}$ University of Michigan, Ann Arbor, MI 48109, USA \\ Correspondence to: Davide Masutti (masutti@vki.ac.be)
}

Received: 14 January 2016 - Revised: 4 July 2016 - Accepted: 5 July 2016 - Published: 1 September 2016

\begin{abstract}
The accuracy of global atmospheric models used to predict the middle/lower thermosphere characteristics is still an open topic. Uncertainties in the prediction of the gas properties in the thermosphere lead to inaccurate computations of the drag force on space objects (i.e. satellites or debris). Currently the lifetime of space objects and therefore the population of debris in low Earth orbit (LEO) cannot be quantified with a satisfactory degree of accuracy. In this paper, the Global Ionosphere Thermosphere Model (GITM) developed at the University of Michigan has been validated in order to provide detailed simulations of the thermosphere. First, a sensitivity analysis has been performed to investigate the effect of the boundary conditions on the final simulations results. Then, results of simulations have been compared with flight measurements from the CHallenging Minisatellite Payload (CHAMP) and Gravity Recovery and Climate Experiment (GRACE) satellites and with existing semiempirical atmospheric models (IRI and MSIS). The comparison shows a linear dependency of the neutral density values with respect to the solar activity. In particular, GITM shows an over-predicting or under-predicting behaviour under high or low solar activity respectively. The reasons for such behaviour can be attributed to a wrong implementation of the chemical processes or the gas transport properties in the model.
\end{abstract}

Keywords. Ionosphere (modelling and forecasting) - magnetospheric physics (solar wind-magnetosphere interactions) - meteorology and atmospheric dynamics (thermospheric dynamics)

\section{Introduction}

The thermosphere is the layer of the Earth's atmosphere directly above the mesosphere and directly below the exosphere (90-600 km altitude). The thermosphere can be described in terms of density, chemical composition, temperature and wind velocity. The thermosphere, largely driven by the solar and geomagnetic activity, shows a strong coupling with the ionosphere and the magnetosphere (Lu et al., 1995). This atmospheric layer is characterised by a high degree of variability. Thermosphere density varies over a wide range of spatial and temporal scales under the influence of the complex interactions between the Earth system and the solar processes. Unfortunately, the measurements of the thermosphere have always been quite sparse and their uncertainties have often introduced a relatively high level of ambiguity. The accurate description of the thermosphere can be a key aspect for the design of a space mission in low Earth orbit (LEO). In particular, the aerodynamic properties impact the lifetime and the attitude control of LEO satellites. In order to accurately predict the satellite orbital decay, for example, a more detailed modelling of the atmosphere density is necessary. Indeed, the neutral air density is affected significantly by the incoming solar radiation and its value can even increase by 2 orders of magnitude during geomagnetic storms as shown by Sutton et al. (2005) and Krauss et al. (2015). During a solar storm event in March 1991 as reported by Sagalyn and Bowhill (1993), a high number of satellites were lost from the NORAD catalogue because their trajectory changed substantially as a result of the increased neutral density and consequent drag increase. This prevented the necessary conjunction analysis between LEO objects, as well as possible eva- 
sive manoeuvres leading to a risk to space assets and astronauts.

Currently, the atmospheric forecasts are based on semiempirical models for the gas neutral composition; among those models there are MSIS-90 (Hedin, 1991), NRLMSISE00 (Picone et al., 2002), DTM (Bruinsma, 2015) and JB2008 (Bowman et al., 2008). These models are finely tuned to match a database of flight measurements, but when applied outside the interpolated ranges they are subjected to large uncertainties in atmosphere density and composition. These uncertainties affect the orbital propagator calculation by leading to an error of several kilometres in the estimated position of LEO satellites (Vallado and Finkleman, 2014). This suggests that physics-based modelling should be used to forecast the state of the upper atmosphere. Such modelling inherently needs a thorough validation.

Among the different physical models available, the general circulation models (GCMs) cover an important field of atmospheric modelling. GCMs like CMAT2 (Harris, 2001), TIEGCM (Richmond et al., 1992), CTIPe (Millward et al., 1996) or GITM (Ridley et al., 2006) require an accurate implementation of the thermosphere phenomena in order to provide validated results. The last of these models, the Global Ionosphere Thermosphere Model (GITM), has been used for this investigation. Neutral density and electron density data available from CHAMP (CHallenging Minisatellite Payload) and GRACE (Gravity Recovery and Climate Experiment) have been compared with the simulations in order to investigate the effect of the solar activity on GITM.

Because of the increasing number of objects in low Earth orbit, the need for an accurate real-time forecasting tool has become critical. In 2016, the QB50 mission will launch one of the biggest CubeSat constellations in order to perform multi-point measurements of the predominant species down to $200 \mathrm{~km}$ altitude (Thoemel et al., 2014). These scientific measurements will enhance the understanding of atmospheric characteristics in the middle and lower thermosphere and they will provide data for a more accurate validation of GCMs.

In Sect. 2, it is presented a brief description of GITM and the flight data used for the validation. The results obtained by comparing the computations and the flight data over a complete range of solar flux values are discussed in Sect. 3. Section 4 is devoted to the conclusions.

\section{Methodology}

\subsection{GITM}

GITM is a three-dimensional Navier-Stokes equation solver that models the Earth's thermosphere and ionosphere system using a spherical coordinate grid. The GITM code solves the coupled continuity, momentum and energy equations of neutrals and ions in a time-marching fashion. For the ions, the

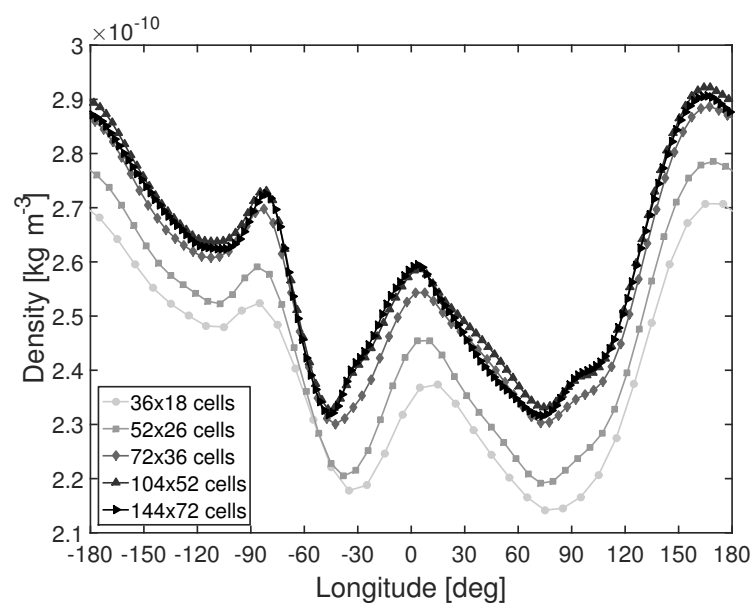

Figure 1. Grid sensitivity analysis at $0^{\circ}$ latitude and $200 \mathrm{~km}$ altitude (long $\times$ lat cells).

velocity variation as a function of time is ignored, so the steady-state ion flow velocity is solved. Moreover, for this model the ion temperature consists of a mixture of the electron and neutral temperatures, and the neutrals are solved for using the Navier-Stokes equations. GITM explicitly solves for the neutral densities of $\mathrm{O}, \mathrm{O}_{2}, \mathrm{~N}\left({ }^{2} \mathrm{D}\right), \mathrm{N}\left({ }^{2} \mathrm{P}\right), \mathrm{N}\left({ }^{4} \mathrm{~S}\right), \mathrm{N}_{2}$ and $\mathrm{NO}$, as well as ion species $\mathrm{O}^{+}\left({ }^{4} \mathrm{~S}\right), \mathrm{O}^{+}\left({ }^{2} \mathrm{D}\right), \mathrm{O}^{+}\left({ }^{2} \mathrm{P}\right)$, $\mathrm{O}_{2}^{+}, \mathrm{N}^{+}, \mathrm{N}_{2}^{+}$and $\mathrm{NO}^{+}$. The simulations are initiated using the neutral/ion densities and temperatures from the MSIS90 and IRI (Bilitza, 2001) semi-empirical models. In general, the results of the simulations become independent of the initial conditions after $72 \mathrm{~h}$ of simulated time. In contrast to other general circulation models of the thermosphere, GITM solves the vertical and horizontal advection in separate equations without assuming any hydrostatic equilibrium. This allows the code to take into account the non-constant gravity in the vertical direction and the Coriolis forces (Deng et al., 2008).

Because GITM 3-D computations are based on a structured spherical grid with adjustable spatial discretisation in longitude and latitude, the accuracy of the results needs to be verified as a function of the grid refinement. A reference case simulation on 19 April 2004 has been run for 4 days ( 3 days of "spin-up" +1 day of simulation). From the grid analysis results shown in Figs. 1-3, it is observed how the grid refinement increases the accuracy of the simulations. This is illustrated, for example, in Fig. 1, where the neutral density values of the atmosphere at an altitude of $200 \mathrm{~km}$ at $0^{\circ}$ latitude are displayed as a function of the longitudinal direction. Even if the density values are confined in a very narrow range between $2 \times 10^{-10}$ and $3 \times 10^{-10} \mathrm{~kg} \mathrm{~m}^{-3}$, the different longitudinal profiles are scattered as a function of the grid refinement. By increasing the number of cells in the mesh, the density profiles tends to converge to similar results. In fact, the relative error between the density profiles with a refinement of $144 \times 72$ cells $\left(2.5^{\circ}\right.$ long $\times 2.5^{\circ}$ lat $)$ and $104 \times 7252$ cells 


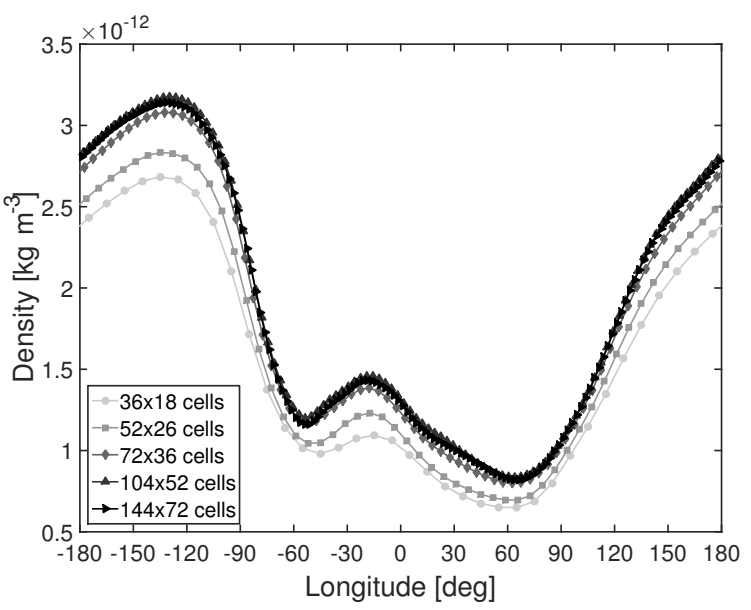

Figure 2. Grid sensitivity analysis at $0^{\circ}$ latitude and $400 \mathrm{~km}$ altitude (long $\times$ lat cells).

$\left(3.46^{\circ}\right.$ long $\times 3.46^{\circ}$ lat) is less than $0.5 \%$, whereas the relative error between the coarser $\left(36 \times 18\right.$ cells or $10^{\circ}$ long $\times 10^{\circ}$ lat $)$ and the finer $\left(144 \times 72\right.$ cells or $2.5^{\circ}$ long $\times 2.5^{\circ}$ lat $)$ grid is $7 \%$. In both cases, the density profiles seem to have peaks and valleys at similar longitudinal locations. With the same grid analysis repeated at an altitude of $400 \mathrm{~km}$ (Fig. 2), the relative error between the coarser and finer grid is increased to almost $19 \%$. However, the relative error between the two most refined grids is as low as $1.2 \%$. The analysis suggests that the quality of the results obtained from GITM simulations are in general grid-dependent below a certain refinement threshold. Because GITM uses a spherical grid for its computations, the cell dimensions change as a function of the altitude for a constant grid refinement. This implies that, for a grid with $144 \times 72$ cells $\left(5^{\circ}\right.$ long $\times 5^{\circ}$ lat $)$, the side length of a squared cell at $200 \mathrm{~km}$ is about $286 \mathrm{~km}$, whereas this value linearly increases to about $295 \mathrm{~km}$ length for an altitude of $400 \mathrm{~km}$.

Figure 3 again shows a grid sensitivity analysis of the density profiles over a meridian at $0^{\circ}$ longitude and $400 \mathrm{~km}$ altitude. It can be seen from also looking at the meridian profiles that the relative difference between the two finer grids $(144 \times 72$ and $104 \times 7252$ cells $)$ is less than $1.5 \%$. Because of the low relative difference observed between the two finer grids in Figs. 1, 2 and 3, it can be assumed that the accuracy of the results will not benefit from a further refinement of the mesh in both longitude and latitude. In order to find a tradeoff between the accuracy of the simulations and the computation time, it was decided to select a grid with $72 \times 36$ cells in longitude and latitude respectively. This grid, used hereafter, allows for results to be obtained with only $3 \%$ difference from the most refined grid in $400 \mathrm{CPU}$ hours instead of 1500 CPU hours (for 4 simulated days). The GITM results, discussed in Sect. 3, have been obtained from 3-D runs with a total of 15 simulated days each ( 3 days of "spin-up" + 12 days

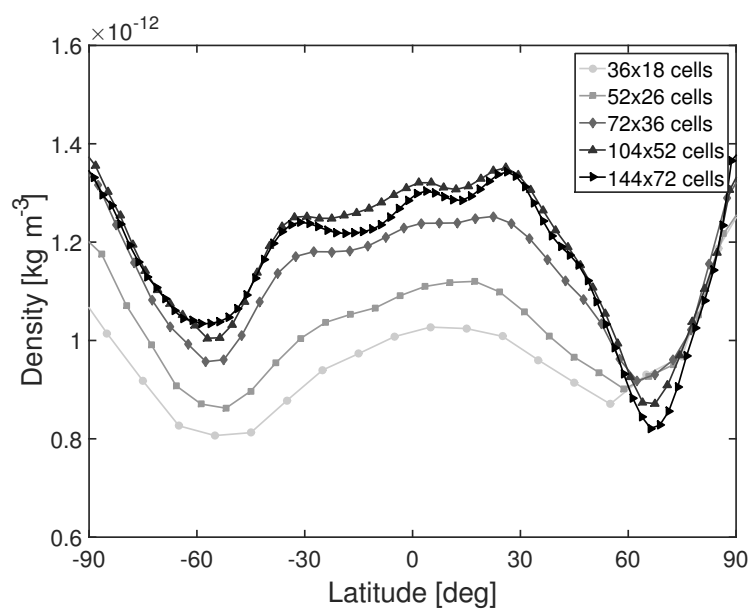

Figure 3. Grid sensitivity analysis at $0^{\circ}$ longitude and $400 \mathrm{~km}$ altitude (long $\times$ lat cells).

of simulation) in order to be fully representative of the model performances.

\subsection{Validation data}

CHAMP is a German small-satellite mission for geoscientific and atmospheric research and applications, managed by the GeoForschungsZentrum (GFZ). Between 2000 and 2010, CHAMP simultaneously generated, for the first time, highprecision gravity and magnetic field measurements. These measurements allowed for the detection of not only the spatial variations in both fields but also their variability with time (Reigber and Schwintzer, 1995). The accelerations on CHAMP are measured by the STAR (Space Three-axis Accelerometer for Research) instrument developed by ONERA (Touboul et al., 1998). Its objective is to measure all nongravitational accelerations of the satellite (drag, solar and Earth radiation pressure) in order to determine the Earth's gravity field from purely gravitational orbit perturbations. The accelerometer measurement principle is based on electrostatic suspension of a proof mass in a cage. The instantaneous position of the proof mass is measured by three capacitive sensors, which permit a determination of the acceleration vector and resulting drag and density. The instrument has a dynamic range of $\pm 1 \times 10^{-4} \mathrm{~m} \mathrm{~s}^{-2}$, a resolution of better than $\pm 3 \times 10^{-9} \mathrm{~m} \mathrm{~s}^{-2}$, and a frequency range of $10^{-1}$ to $10^{-4} \mathrm{~Hz}$. The STAR instrument is positioned at the centre of gravity of CHAMP to minimise the effect of rotational accelerations and gravity gradients on the measurements.

Data derived from STAR can be used to calculate the neutral density with an uncertainty level of $10-15 \%(1 \sigma)$ for moderate geomagnetic activity (Bruinsma et al., 2004). Sutton (2009) shows an overestimation of the CHAMP density values of about $20 \%$ when compared to the HASDM semiempirical model. Doornbos (2012) observed a similar overestimation when comparing the same semi-empirical model 


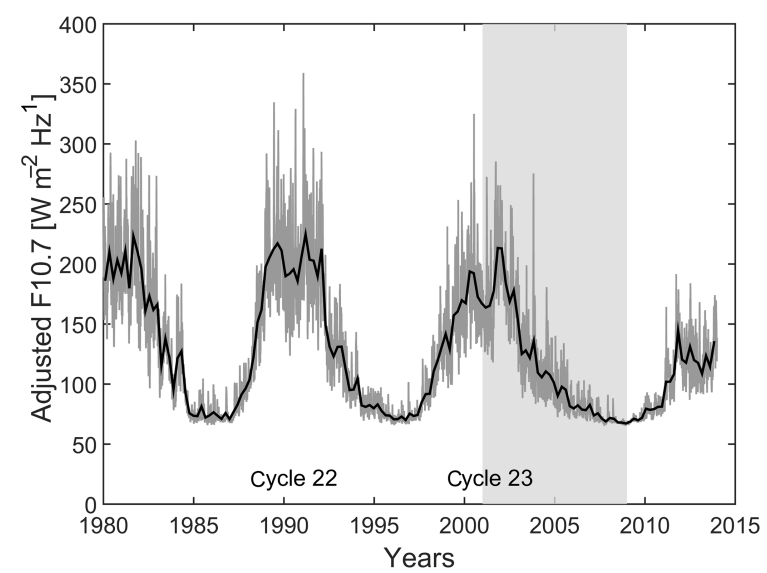

Figure 4. Daily (grey line) and 81-day-averaged (black line) solar flux received on Earth at $10.7 \mathrm{~cm}$ wavelength between 1980 and 2015. The grey region represents the selected investigation period.

data with CHAMP densities processed with a different retrieval algorithm. Both Sutton (2009) and Doornbos (2012) conclude that the bias in the density values is caused by the approximations in the modelling of the external satellite geometry and the aerodynamic interaction of the gas with the spacecraft walls. The design of the CHAMP satellite, with its complex elongated shape and protruding instruments, antennae and baffles, is not ideal for the purpose of density measurements.

CHAMP has also a planar Langmuir probe (PLP) in order to measure the atmosphere electron density with an uncertainty within $10 \%(1 \sigma)$ (Liu et al., 2007). The PLP provides measurements of the electron density and temperature every $15 \mathrm{~s}$ for $1 \mathrm{~s}$ in the voltage sweep mode. Thus, CHAMP data from the Sutton (2009) database v2.2 are available every $15 \mathrm{~s}$, which corresponds to a spatial resolution of about $115 \mathrm{~km}$ considering a satellite velocity of $7.6 \mathrm{~km} \mathrm{~s}^{-1}$. Further details of the PLP design and performance are summarised by Cooke et al. (2003) and McNamara et al. (2007). In the present paper, novel comparisons have been performed between numerical models and electron density data from CHAMP.

On top of CHAMP data, GITM simulations have also been compared with GRACE flight data. GRACE was launched in 2002 and consists of two almost identical satellites which follow each other on the same orbit at a distance of about $220 \mathrm{~km}$ (Kang et al., 2006). The orbits are circular polar, with an initial altitude of $485 \mathrm{~km}$ at launch (near solar maximum 23) and an inclination of $89^{\circ}$. The design of the GRACE satellites is similar to the CHAMP satellite. Indeed, these twin satellites contain a GPS receiver, an extremely precise accelerometer and star tracker cameras. In addition, each of the GRACE satellites carries a K-band horn directed at the other satellite for inter-communication. Like CHAMP, these satellites have the main objective of investigating Earth's gravity field and its small variations.

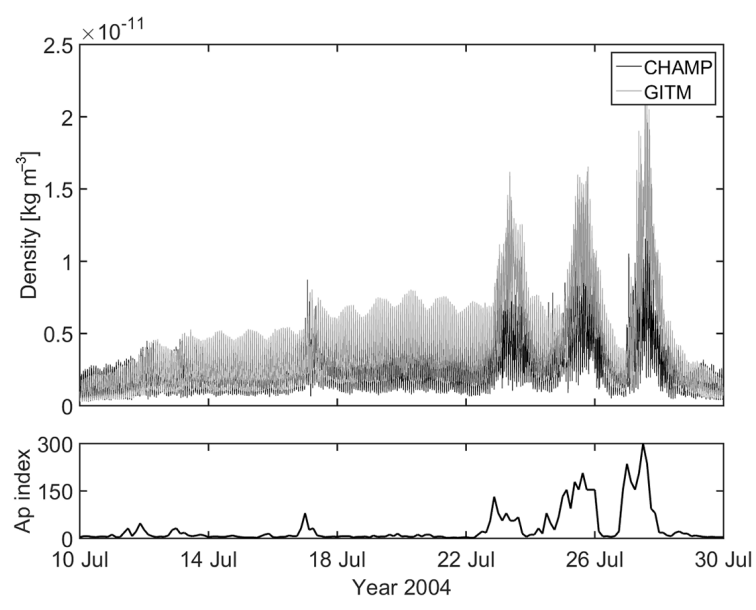

Figure 5. Effect of the geomagnetic storminess on GITM and CHAMP neutral densities from 10 to 30 July 2004.

Table 1. Boundary conditions of the simulations.

\begin{tabular}{lcrr}
\hline & Date (DD/MM/YYYY) & $\begin{array}{r}\mathrm{F} 10.7 \\
\left(\mathrm{~W} \mathrm{~m}^{-2} \mathrm{~Hz}^{-1}\right)\end{array}$ & Ap index \\
& & $233.1 \pm 24.5$ & $9 \pm 7$ \\
\hline Case a & $18 / 12 / 2001$ & $145.6 \pm 5.2$ & $7 \pm 4$ \\
Case b & $15 / 06 / 2002$ & $112.9 \pm 8.2$ & $12 \pm 8$ \\
Case c & $28 / 08 / 2003$ & $100.3 \pm 9.9$ & $8 \pm 5$ \\
Case d & $10 / 04 / 2004$ & $77.4 \pm 1.8$ & $5 \pm 4$ \\
Case e & $10 / 10 / 2005$ & $66.7 \pm 0.8$ & $5 \pm 4$ \\
Case f & $24 / 10 / 2008$ & & \\
\hline
\end{tabular}

\section{Results}

\subsection{Sensitivity to solar and geomagnetic variability}

The solar flux F10.7 is a good proxy to describe the daily solar activity; Fig. 4 shows the cyclic behaviour of this index between the years 1980 and 2015. The grey line in Fig. 4 shows the daily variation in the F10.7 proxy, whereas the black line represents the 81 -day-averaged variation. The grey area indicates the corresponding solar activity period during the descending phase of the 23rd solar cycle, where the F10.7 values are ranging from 66.7 to $233.1 \mathrm{~W} \mathrm{~m}^{-2} \mathrm{~Hz}^{-1}$. Table 1 summarises the boundary conditions of the cases under investigation in this paper. These specific simulation windows have been selected in periods of low geomagnetic activity $(A p<30)$ in order to avoid any geomagnetic storm that could interfere with the study. Figure 5 shows the impact of a solar storm on CHAMP flight data and GITM simulations. Three distinct storms (Ap $>=150)$ impacted the thermosphere between 22 and 27 July 2004. Both the data and simulations show an increase of the neutral density by almost 1 order of magnitude.

The neutral density of the GITM simulations from Table 1 has been averaged over a period of 12 days and presented as a function of altitude, latitude and local time in order to extract 


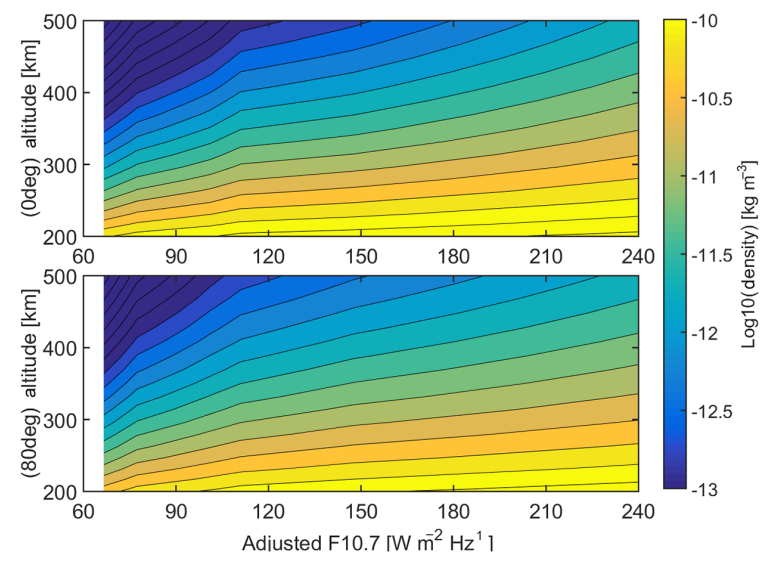

Figure 6. Logarithmic variation in the atmosphere density at $0^{\circ}$ and $80^{\circ} \mathrm{N}$ latitude and 02:00 LT as a function of altitude and adjusted solar flux (F10.7).

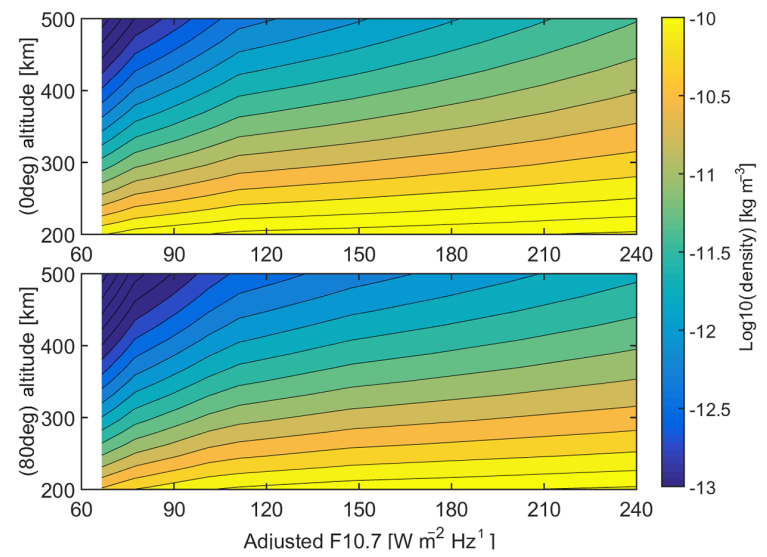

Figure 7. Logarithmic variation in the atmosphere density at $0^{\circ}$ and $80^{\circ} \mathrm{N}$ latitude and 14:00 LT as a function of altitude and adjusted solar flux (F10.7).

the long term effect of the solar flux input. Figures 6 and 7 show the effect of the F10.7 values on the simulated density in the thermosphere at $0^{\circ}$ and $80^{\circ} \mathrm{N}$ latitude as a function of altitude at 02:00 and 14:00 local time (LT) respectively. As can be observed from Fig. 6, the sensitivity of the atmosphere density to the solar flux is increasing with the increasing altitude. At about $150 \mathrm{~km}$ altitude and below there is a small effect of the Sun's activity, and the air density can be considered almost independent of the energy radiated by the Sun. However, at an altitude of $400 \mathrm{~km}$, the air density value can increase by 2 orders of magnitudes between low and high solar activity (i.e. 66.7 to $233.1 \mathrm{~W} \mathrm{~m}^{-2} \mathrm{~Hz}^{-1}$ respectively). The comparison between Figs. 6 and 7 shows that the increment of density between low and high solar activity is dependent on both local time and latitude. In particular, the density increase is slightly bigger at $80^{\circ} \mathrm{N}$ with respect to $0^{\circ}$ (at constant local time) and on the nightside with respect to the dayside (at constant latitude). Because of this large variation

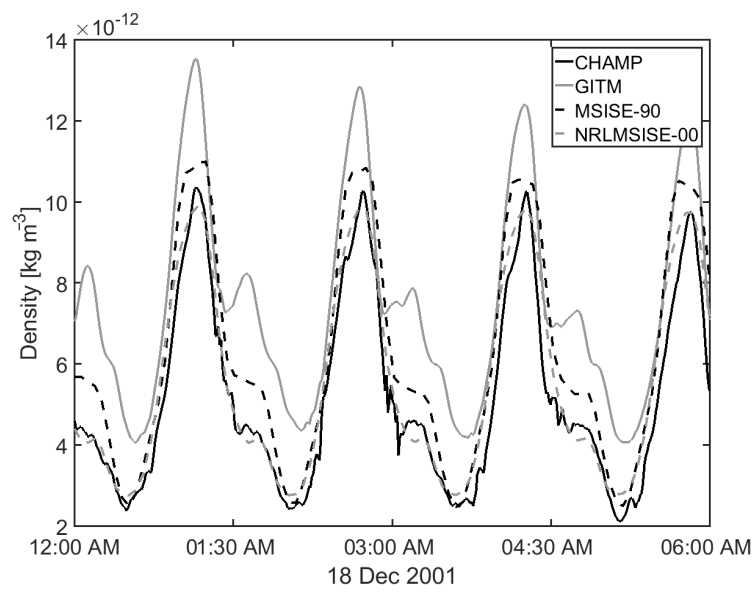

Figure 8. Comparison of neutral density as a function of time from GITM simulations (solid black line), CHAMP flight data (solid grey line) and semi-empirical models (dashed line).

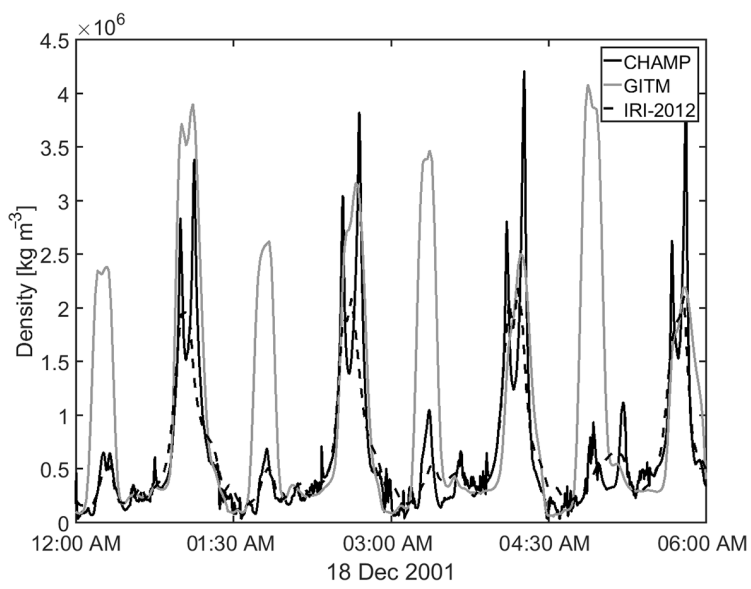

Figure 9. Comparison of electron number density as a function of time from GITM simulations (solid black line), CHAMP flight data (solid grey line) and a semi-empirical model (dashed line).

in density in the mid-lower thermosphere, satellites in low Earth orbit must increase their orbital altitude to take into account the increased aerodynamic drag during periods of high solar activity. An example is the International Space Station (Zhukov et al., 2012).

\subsection{Comparison with flight data}

The results of the GITM simulations are compared with neutral densities and electron densities measured by CHAMP and GRACE. As an example, the trend of the simulations and the flight data is presented in Figs. 8 and 9 as a function of time (only $6 \mathrm{~h}$ ) for the neutral and the electron density respectively. As can be observed in Fig. 8, the density shows a sinusoidal-like behaviour typical of the day-night sequence. The higher peaks correspond to a high density over the Equator on the dayside, whereas the valleys correspond to the pas- 


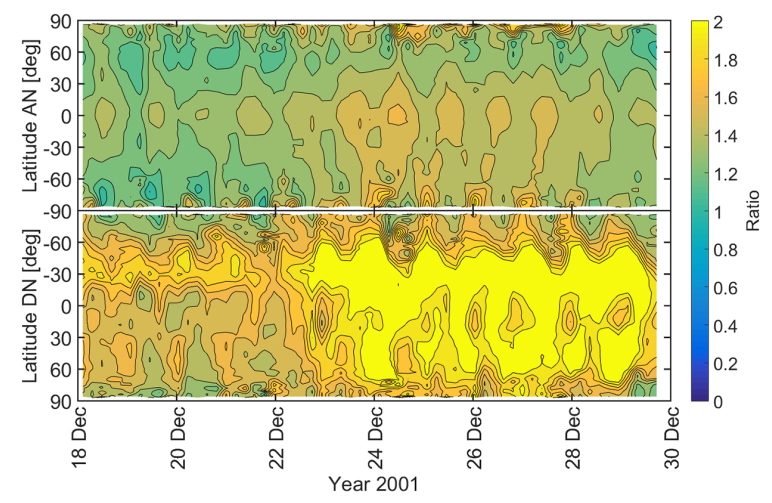

Figure 10. Density ratio (GITM/CHAMP) as a function of time and latitude for "case a" (local time of ascending node, LTAN $=15: 10$; local time of the descending node, $\operatorname{LTDN}=03: 10$; $h_{\text {apogee }}=469 \mathrm{~km} ; h_{\text {perigee }}=405 \mathrm{~km}$ ).

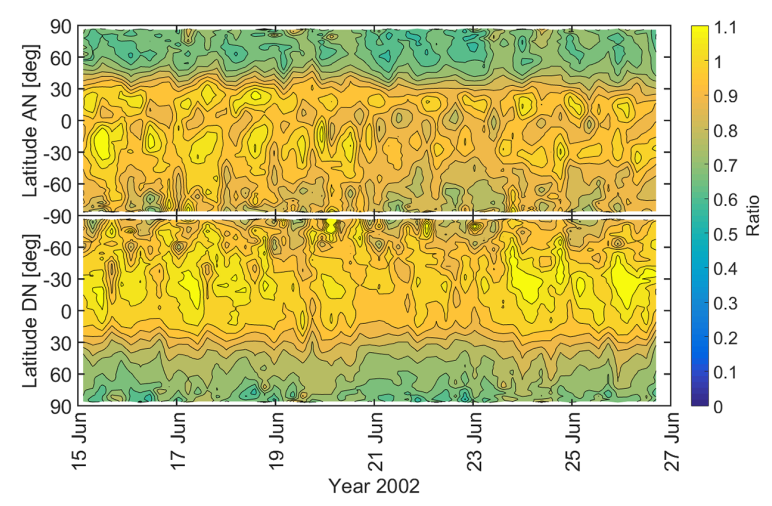

Figure 11. Density ratio (GITM/CHAMP) as a function of time and latitude for "case b" (LTAN $=22: 50 ; \operatorname{LTDN}=10: 50$; $h_{\text {apogee }}=449 \mathrm{~km} ; h_{\text {perigee }}=400 \mathrm{~km}$ ).

sage over the South Pole on the nightside. The agreement between the flight data and the semi-empirical models is very good, especially with the NRLMSISE-00 model. However, a marked overestimation of the order of $50 \%$ can be observed from the comparison between the GITM simulations and the flight data.

In Fig. 9 the electron densities obtained from CHAMP, the GITM simulations and the semi-empirical model are compared. The agreement with the flight data and the IRI-2012 model is good, and again less good agreement with the GITM simulations is observed. In particular, on the nightside, the simulations around the Equator show a highly over-predicted electron density with respect to flight data. The reason for such an overestimation can be attributed to the missing divergence term in the electron continuity equation in GITM. Such a missing term together with the missing electrodynamic model does not allow GITM to account for the proper electron downwelling which causes accumulations of electrons on the nightside in the equatorial region.

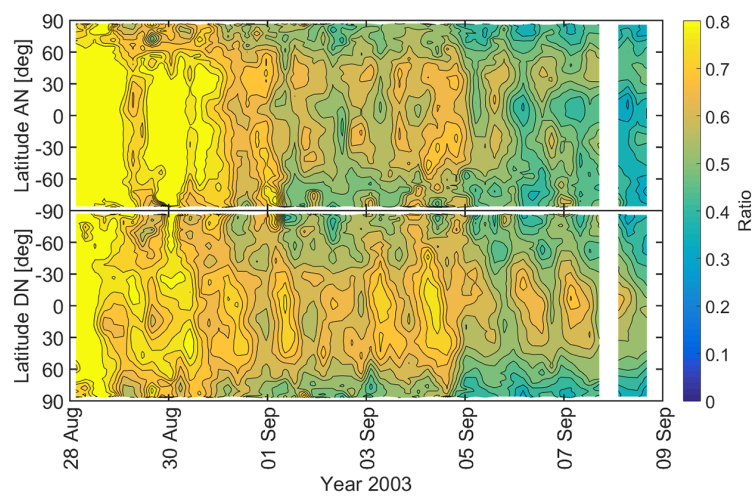

Figure 12. Density ratio (GITM/CHAMP) as a function of time and latitude for "case c" $(\mathrm{LTAN}=06: 44$; $\mathrm{LTDN}=18: 44$; $h_{\text {apogee }}=423 \mathrm{~km} ; h_{\text {perigee }}=392 \mathrm{~km}$ ).

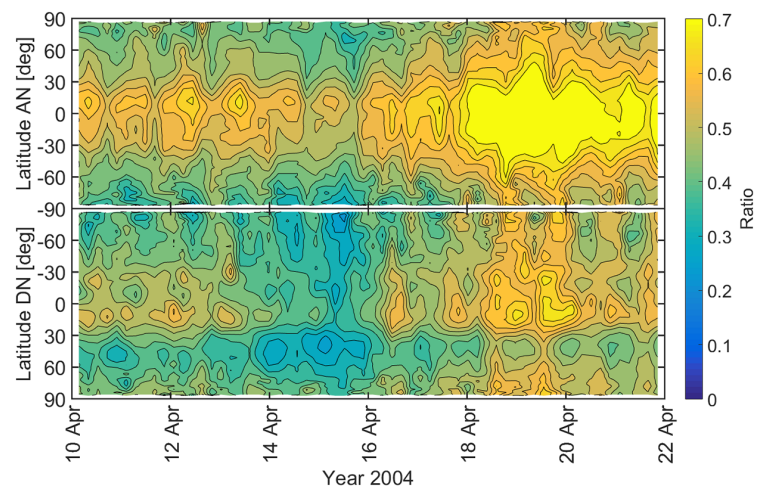

Figure 13. Density ratio (GITM/CHAMP) as a function of time and latitude for "case d" (LTAN $=10: 01$; $L T D N=22: 01$; $h_{\text {apogee }}=410 \mathrm{~km} ; h_{\text {perigee }}=378 \mathrm{~km}$ ).

Figures 10-15 show a comprehensive comparison between GITM simulations and CHAMP neutral densities as a function of latitude and time for all cases presented in Table 1. All figures represent the ratio between GITM-simulated density and CHAMP flight data. A density ratio value of 1 corresponds to a good validation between the simulations and the experiments, whereas values larger or smaller than 1 indicate that the GITM density is over-predicted or under-predicted with respect to CHAMP data. The ratio values are shown as a function of time and latitude for the ascending/descending branch of the orbit. Local times of ascending/descending nodes and orbit altitude are given for each case under investigation. In general, Figs. 10-15 show that GITM simulations are consistent in time and they are representative of the behaviour of the model under the different solar activity periods.

For all cases (a-f), GITM simulations present a different prediction behaviour over the poles than over the Equator. Over the poles the computed neutral density tends always to under-predict the flight data with respect to the predictions at the Equator. This is true both for the ascending/descending 


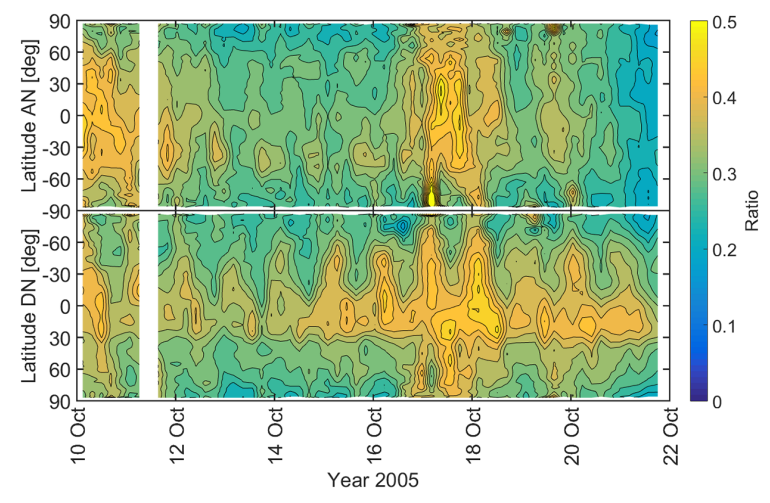

Figure 14. Density ratio (GITM/CHAMP) as a function of time and latitude for "case e" (LTAN $=07: 38 ;$ LTDN $=19: 38$; $\left.h_{\text {apogee }}=379 \mathrm{~km} ; h_{\text {perigee }}=350 \mathrm{~km}\right)$.

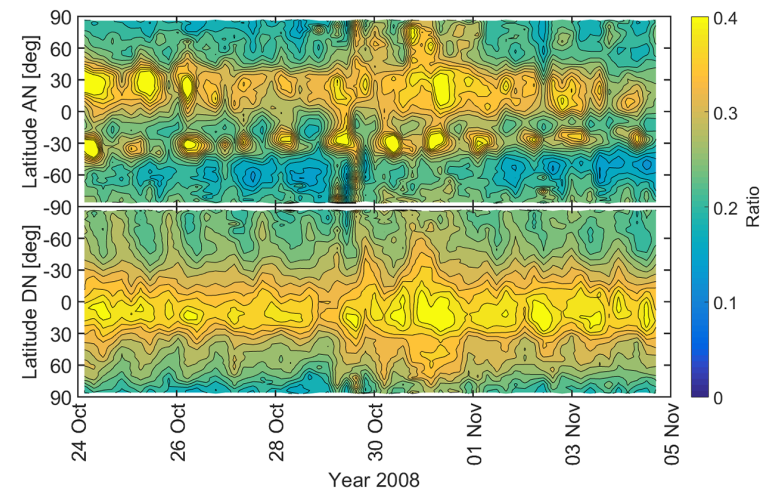

Figure 15. Density ratio (GITM/CHAMP) as a function of time and latitude for "case f" (LTAN $=01: 06 ;$ LTDN $=13: 06$; $h_{\text {apogee }}=353 \mathrm{~km} ; h_{\text {perigee }}=323 \mathrm{~km}$ )

branch of the orbit. Figures 10, 11, 13 and 15 show that, around $\pm 30^{\circ}$ latitude, there are two distinct zones of high relative ratio values with respect to the rest of the nightside orbit branch. These high relative ratio values correspond to the under-prediction or the over-prediction of the equatorial mass anomaly (EMA) by GITM. This phenomenon, generated by the equatorial electrojet (EEJ) and recently described by Liu et al. (2005) using CHAMP data, creates an accumulation of density between 15 and $30^{\circ}$ latitude poleward with respect to the magnetic equator. The interaction between the Earth magnetic field and the ions transported by the EEJ causes a Lorentz lift-off mechanism that pushes ions and electrons to higher altitudes over the magnetic equator. When the particles reach the maximum altitude, they fall down and generate an accumulation of density with a fountain-like shape. Because of the experimental nature of the electrodynamics model in GITM (i.e. dynamo modelling), it was decided to disable such a feature during the investigation so that the EMA phenomenon cannot be correctly modelled in the current simulations. As can be seen from Figs. 12 and 14, when the orbit crosses the ascending/descending nodes at

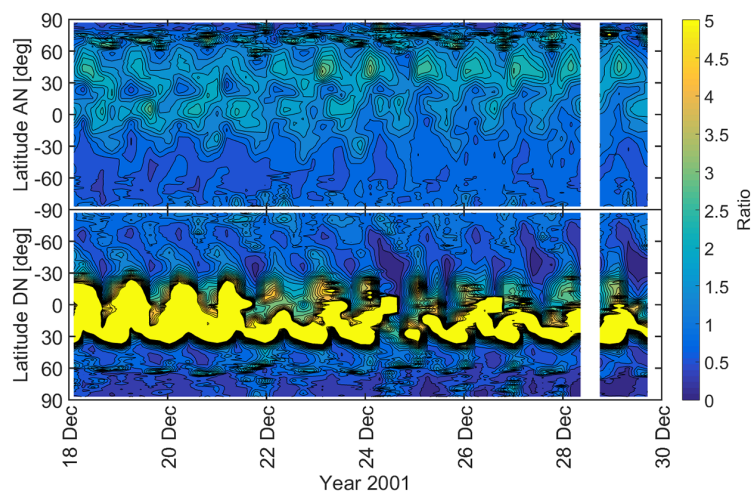

Figure 16. Electron density ratio (GITM / CHAMP) as a function of time and latitude for "case a" (LTAN $=15: 10$; LTDN $=03: 10)$.

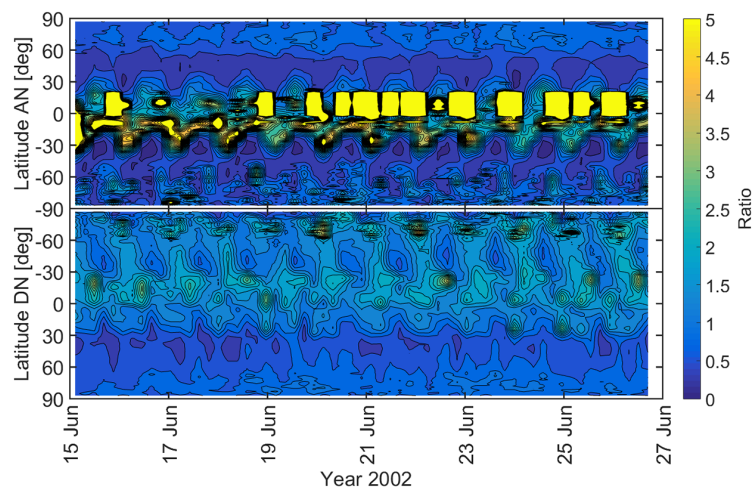

Figure 17. Electron density ratio (GITM / CHAMP) as a function of time and latitude for "case b" (LTAN = 22:50; LTDN = 10:50).

dawn and dusk respectively, the ratio values of the ascending/descending branch of the orbit are quite similar to each other. Moreover, for both cases the under/over-prediction of the EMA is visible during the ascending branch as well as the descending branch of the orbit.

Figures 16-21 represent the comparison between GITM simulations and CHAMP electron number densities as a function of latitude and time for all cases presented in Table 1 . The values shown in the figures are again the ratios between the simulations and the flight data. As discussed above for Fig. 9, in the comprehensive analysis shown in Figs. 16-21 it can be observed that GITM simulations are unable to correctly predict the electron density on the nightside at $\pm 30^{\circ}$ over the Equator. All cases indicate that the ratio between the simulations and the flight data is equal to 5 (or higher) on the nightside. Again, in Figs. 16-21 it can be observed that the results are consistent over time and therefore representative of the behaviour of the model. The comparison between the simulated and measured electron densities shows similar features to those in the analysis carried out for the neutral densities. In particular, on the dayside the EMA phenomenon is still visible at $\pm 30^{\circ}$ poleward over the magnetic equator, where the typical double peak is either over- 


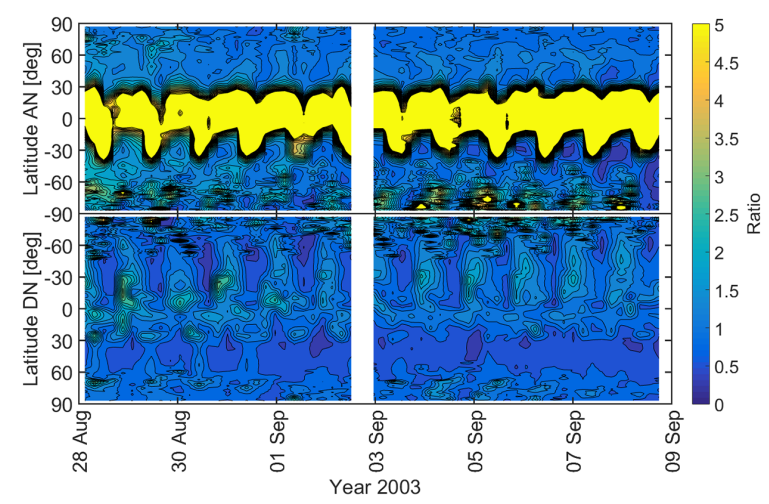

Figure 18. Electron density ratio (GITM / CHAMP) as a function of time and latitude for "case c" (LTAN $=06: 44 ; \mathrm{LTDN}=18: 44)$.

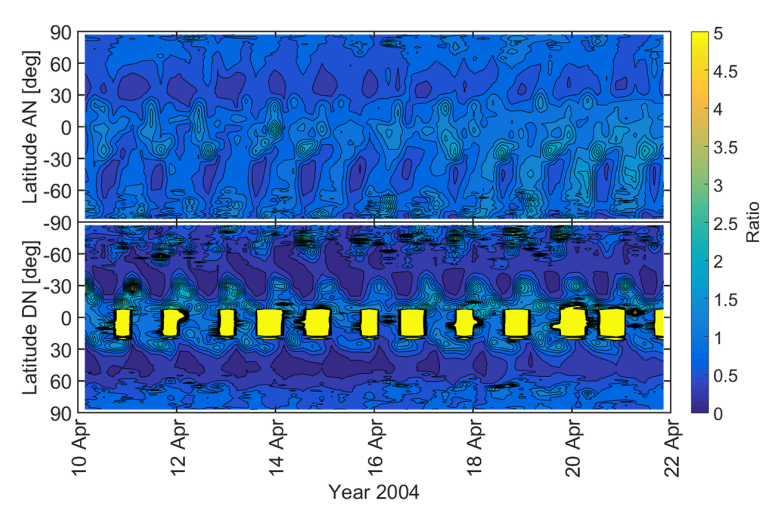

Figure 19. Electron density ratio (GITM / CHAMP) as a function of time and latitude for "case d" (LTAN = 10:01; LTDN = 22:01).

or underestimated by the simulations because of the missing electrodynamic model. The analysis over the poles and over the Equator shows again a different behaviour of the model in the two regions. In general, it can be observed that the ratios over the poles are closer to a value of 1 for all cases under investigation, whereas the ratio values over the Equator are more scattered.

\subsection{Effect of the solar activity}

Figure 22 summarises the behaviour on GITM for a wide spectrum of solar flux values. The ratios between the measured and simulated neutral densities are given as a function of the F10.7 index and for CHAMP and GRACE satellites. Every point shown in Fig. 22 is the result of an orbital average of the ratios between the simulations and the flight data. Figure 22 indicates that there is a linear dependency between the accuracy of the GITM simulations and the solar activity. In particular, the simulated neutral densities are underestimating the flight data by a factor of 0.4 during low solar activity $\left(85 \mathrm{~W} \mathrm{~m}^{-2} \mathrm{~Hz}^{-1}\right)$, whereas they are overestimating the flight data by a factor of 1.7 during high solar activity $\left(260 \mathrm{~W} \mathrm{~m}^{-2} \mathrm{~Hz}^{-1}\right)$. The density simulated by GITM

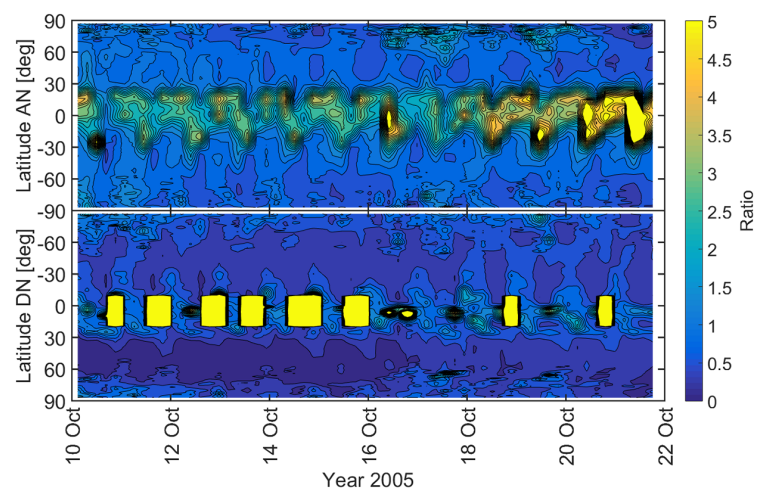

Figure 20. Electron density ratio (GITM / CHAMP) as a function of time and latitude for "case e" (LTAN =7:38, LTDN = 19:38).

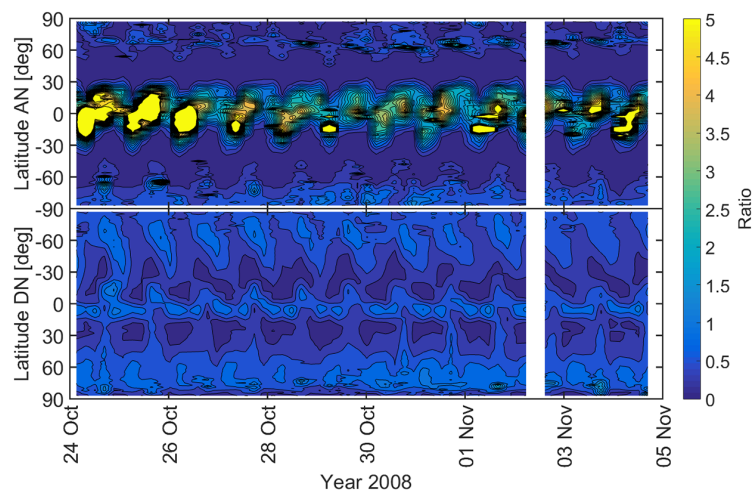

Figure 21. Electron density ratio (GITM / CHAMP) as a function of time and latitude for "case f" (LTAN = 1:06, LTDN = 13:06).

agrees well (1:1 ratio) with the flight measurement only for F10.7 values between 150 and $170 \mathrm{~W} \mathrm{~m}^{-2} \mathrm{~Hz}^{-1}$ (i.e. during medium solar activity).

Figure 23 shows the results obtained from the comparison between the simulated and measured electron densities as a function of the solar flux. The results shown are affected by a high degree of scattering, in particular on the nightside. As explained in Sect. 3.2, a wrong implementation of the electron continuity equation in GITM is the reason for a high density of electrons on the nightside. Because of such an implementation, electrons are not correctly downwelling to lower altitudes on the nightside, which is the reason for their accumulation and the extremely high ratio on the nightside. However, the results from the comparison on the dayside, even if they are still affected by a marked scattering, again show a linear dependency with the solar flux. In conclusion, the sensitivity of GITM to the solar flux is higher for the neutral density (solid line) than for the electron density (dashed line).

An investigation of the reasons for such sensitivity is currently ongoing and some preliminary causes are presented here. 


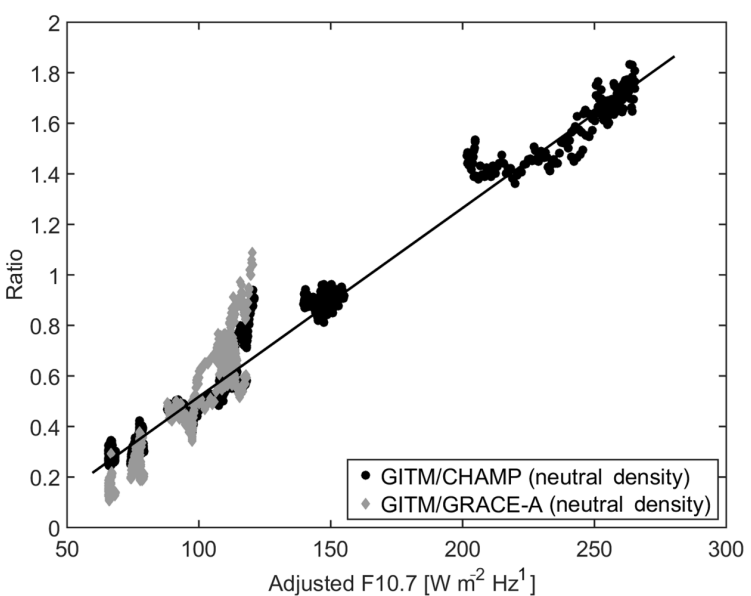

Figure 22. Effect of the solar activity on a large dataset of GITM simulations in comparison with CHAMP and GRACE neutral densities. The solid line represents the linear interpolation of all neutral density ratios.

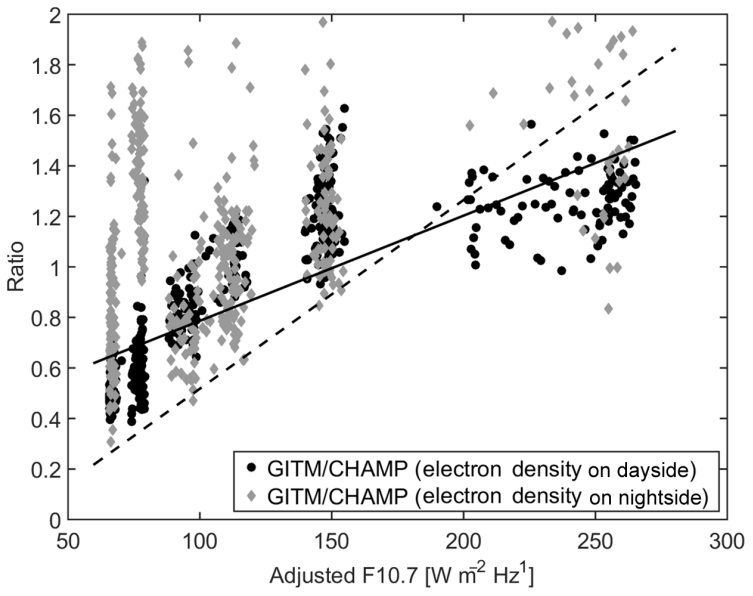

Figure 23. Effect of the solar activity on a large dataset of GITM simulations in comparison with CHAMP electron number density. The solid line represents the linear interpolation of all neutral density ratios (from Fig. 22). The dashed line represents the linear interpolation of dayside-only electron number density ratios.

- The transport properties, namely the dynamic viscosity and the thermal conductivity, are not correctly implemented in GITM. For example, the viscosity model presents a wrong trend as a function of temperature if compared to a simple Sutherland formulation. Nevertheless, a preliminary comparison by (Giulioni, 2016) between neutral densities computed with GITM using the original viscosity model and a Sutherland-based model shows a change of less than $5 \%$ in density. The latter comparison suggests that the effect of the viscosity model in GITM is negligible; nevertheless, the effect of a different thermal conductivity model is yet to be investigated.

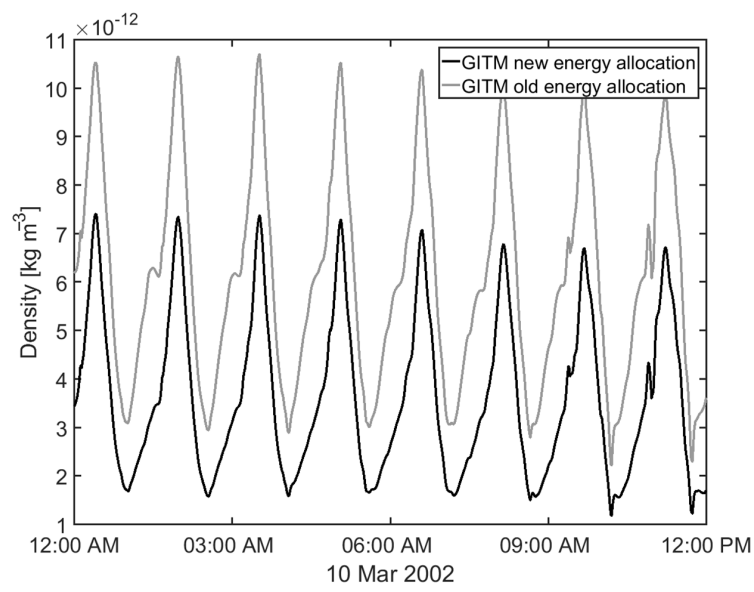

Figure 24. Effect of the new and old allocation of the exothermic excess energy on the simulated density. The density is simulated along the CHAMP trajectory as an example.

- In the version of GITM used in this paper the photoelectron heating is modelled through a semi-empirical law controlled by a fixed photoelectron heating efficiency. This heating coefficient is known to have an important bias effect on the simulations as explained by Burrell et al. (2015). Its value is in fact obtained from data comparison because of the lack of flight measurements. In the last version of GITM, described in the paper by Zhu et al. (2016), the photoelectron efficiency is now treated with an improved formulation that takes into account a heating efficiency as a function of the altitude.

- The coefficients for the chemical reaction rates implemented in GITM are mainly taken from Rees (1989) and Schunk and Nagy (2009). A number of these rates are given as constant, while others are specified as a function of the electron temperature or the mean between the ion and the neutral temperature. Recently, Richards and Voglozin (2011) re-examined the photochemical reactions in the ionosphere and presented a literature survey of the latest chemical reactions with updated coefficient rates in the upper atmosphere. An appreciable difference can be found by comparing the reactions in the paper of Richards and Voglozin (2011) and chemical processes implemented in GITM.

- In GITM, all of the excess energy from the exothermic chemical processes is allocated into the neutrals and none into the ions. This causes an overheating of the model especially in times of large ionisation rates (i.e. with large F10.7 values). As explained by Rees (1989), this approach is not correct because the excess energy should be partitioned between the products of the chemical reaction with an inverse proportion to their masses. Figure 24 presents the results of a preliminary investigation on the effect of the different energy allocations on 


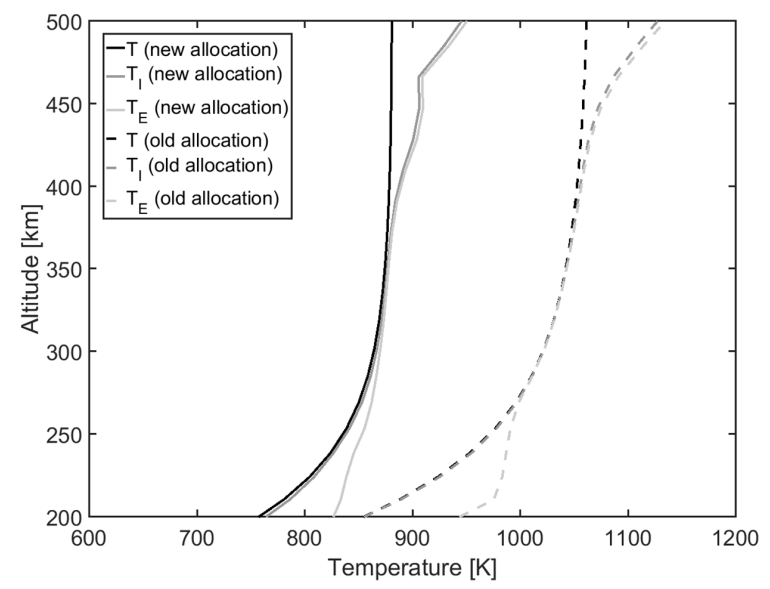

Figure 25. Effect of the new (solid lines) and old (dashed lines) allocation of the exothermic excess energy on the neutral, ion and electron temperature respectively (date: 10 March 2002, 00:00; $0^{\circ}$ latitude and $0^{\circ}$ longitude).

the simulated density. Figure 24 shows the comparison of the simulated densities along the CHAMP trajectory using the new (following Rees, 1989) and the old (original GITM implementation) allocation of the exothermic excess energy. Results show that by allocating the excess energy only in the neutral temperature energy equation, an artificial heating of the model is generated, increasing the density by almost $70 \%$ with respect to the implementation suggested by Rees (1989). This artificial heating is also shown in Fig. 25, where the vertical profiles of all temperatures (neutral, ion and electron) obtained with the original energy allocation are in general $10-25 \%$ higher with respect to the same temperatures obtained using the new energy allocation.

- It is found by Giulioni (2016) that the NO cooling rates computed in GITM are at least 2 times higher with respect to the measured cooling rates by the TIMED/SABER instrument. The reasons for such discrepancy could be attributed to not only a wrong concentration of $\mathrm{NO}$ or $\mathrm{O}$ in the simulations but also incorrect modelling of the cooling mechanism and the related reaction rate coefficients. To further conclude on the NO cooling, the implementation in GITM must be compared to the most recent work in this field presented by Hwang et al. (2003).

In general, Pawlowski and Ridley (2009) showed that, using the broad published values of thermal conductivity, NO cooling rates and other parameters, there is a significant variation in the temperatures and densities derived from the model. This study shows that the default parameters in GITM cause the model to be too sensitive to F10.7 when compared to the mass density. The results presented by Giulioni (2016) using an improved chemistry module (which includes both the updated reaction rates and the corrected excess energy allocation) show that the neutral densities computed with the new module are lower with respect to the same densities simulated with the original implementation of the chemistry module. Giulioni (2016) also shows the performances of the new chemistry module over a limited range of solar activities and observes that the sensitivity of GITM to the F10.7 solar flux is not yet fixed.

\section{Conclusions}

From a grid sensitivity study on GITM a trade-off between the accuracy of the simulations and the computation time has been found. A grid with $72 \times 36$ cells $\left(5^{\circ} \times 5^{\circ}\right)$ in longitude and latitude respectively allows for results to be obtained with only $3 \%$ difference from the most refined grid $\left(144 \times 72\right.$ cells or $\left.2.5^{\circ} \times 2.5^{\circ}\right)$ in a quarter of the computation time (1500 vs. 400 CPU hours).

The comprehensive validation of the GITM simulations and the flight data for both neutral densities and electron number densities confirms the consistency of the results during the different solar activity periods investigated here. The comparison shows the predicting capabilities of GITM, indicating that in general there is a relative under-prediction of the neutral density over the poles. The same comparison also shows that, without the electrodynamic model enabled (i.e. dynamo model), GITM is unable to correctly model the EMA, and this is visible especially on the nightside, where the comparison shows two distinct zones of under/overprediction at $\pm 30^{\circ}$ poleward with respect to the magnetic equator.

It is also found that there is a clear dependency between the GITM bias and the solar activity. In particular, this dependency shows that the ratio between the simulations and the flight measurements is a linear function of the solar flux F10.7. From the data presented it is possible to observe that GITM is underestimating the flight data by a factor of 0.4 during periods of low solar activity $\left(85 \mathrm{~W} \mathrm{~m}^{-2} \mathrm{~Hz}^{-1}\right.$ ), whereas it is overestimating the flight data by almost a factor of 2 during periods of high solar activity $\left(260 \mathrm{~W} \mathrm{~m}^{-2} \mathrm{~Hz}^{-1}\right)$. The simulated neutral densities agree well with the flight measurements only for F10.7 values between 150 and $170 \mathrm{~W} \mathrm{~m}^{-2} \mathrm{~Hz}^{-1}$. The results also show that GITM has a different sensitivity to the solar flux variation for the neutral density and the electron density respectively. In particular, the electron number density bias is less sensitive to a change in the solar flux, and the results have a lower bias with respect to the flight data on the dayside.

There are many reasons that could explain the high sensitivity of GITM to the solar activity. An ongoing study at the University of Michigan and the von Karman Institute is addressing this topic, and the answer is not currently known. Schemes underlying GITM such as the transport properties 
modelling and the chemical reaction rates are the suspected causes.

\section{Code availability}

The GITM code is a property of the University of Michigan and cannot be distributed.

\section{Data availability}

The results from the GITM simulations are publicly available. Because of the considerable amount of data (several gigabytes), the results can only be obtained on request from the main author. All the flight data used to compare with the simulations are third-party data (CHAMP Density v2.2: http://sisko.colorado.edu/sutton/ data/ver2.2/champ/density/; CHAMP Electron Density: http: //isdc-old.gfz-potsdam.de/; GRACE Density v2.2: http:// sisko.colorado.edu/sutton/data/ver2.2/grace/density/).

Author contributions. Davide Masutti prepared the manuscript with the contribution of all co-authors. In particular, Gunther March collected the data and prepared the preliminary simulations. Aaron Ridley provided continuous support with the GITM code and the simulations. Jan Thoemel reviewed the work.

Acknowledgements. The research leading to these results has received funding from the European Union's Seventh Framework Programme (FP7) under REA grant agreement no. 284427. Support for the University of Michigan is from the National Science Foundation (grant ATM1242839).

The topical editor, C. Jacobi, thanks two anonymous referees for help in evaluating this paper.

\section{References}

Bilitza, D.: International Reference Ionosphere 2000, Radio Sci., 36, 261-275, doi:10.1029/2000RS002432, 2001.

Bowman, B. R., Tobiska, W. K., Marcos, F., Huang, C. Y., Lin, C. S., and Burke, W. J.: A New Empirical Thermospheric Density Model JB2008 Using New Solar and Geomagnetic Indices, in: Astrodynamics Specialist Conference, Honolulu, Hawaii, 18-21 August 2008, AIAA 2008-6438, 2008.

Bruinsma, S.: The DTM-2013 thermosphere model, J. Space Weather Space Clim., 5, A1, doi:10.1051/swsc/2015001, 2015.

Bruinsma, S., Tamagnan, D., and Biancale, R.: Atmospheric densities derived from CHAMP/STAR accelerometer observations, Planet. Space Sci., 52, 297-312, doi:10.1016/j.pss.2003.11.004, 2004.

Burrell, A., Goel, A., Ridley, A., and Bernstein, D.: Correction of the photoelectron heating efficiency within the global ionosphere-thermosphere model using Retrospective Cost Model Refinement, J. Atmos. Sol.-Terr. Phy., 124, 30-38, doi:10.1016/j.jastp.2015.01.004, 2015.
Cooke, D. L., Turnbull, W., Roth, C., Morgan, A., and Redus, R.: Ion Drift-Meter Status and Calibration, in: First Champ Mission Results for Gravity, Magnetic, and Atmospheric Studies, Springer, Berlin, 212-219, doi:10.1007/978-3-540-38366-6_31, 2003.

Deng, Y., Richmond, A. D., Ridley, A. J., and Liu, H.-L.: Assessment of the non-hydrostatic effect on the upper atmosphere using a general circulation model (GCM), Geophys. Res. Lett., 35, L01104, doi:10.1029/2007GL032182, 2008.

Doornbos, E.: Thermospheric Density and Wind Determination from Satellite Dynamics, Springer-Verlag, Berlin Heidelberg, doi:10.1007/978-3-642-25129-0, 2012.

Giulioni, L.: Accurate modeling of the gas properties in the thermosphere under high and low solar activity conditions, Project report rm 2016-12, von Karman Institute, Sint-Genesius-Rode, Belgium, 2016.

Harris, M. J.: A new coupled middle atmosphere and thermosphere circulation model: Studies of dynamic, energetic and photochemical coupling in the middle and upper atmosphere, $\mathrm{PhD}$ thesis, University College London, UK, 2001.

Hedin, A. E.: Extension of the MSIS Thermospheric Model into the Middle and Lower Atmosphere, J. Geophys. Res., 96, 1159, doi:10.1029/90JA02125, 1991.

Hwang, E. S., Castle, K. J., and Dodd, J. A.: Vibrational relaxation of $\mathrm{NO}(v=1)$ by oxygen atoms between 295 and $825 \mathrm{~K}$, J. Geophys. Res.-Space, 108, 1109, doi:10.1029/2002JA009688, 2003.

Kang, Z., Tapley, B., Bettadpur, S., Ries, J., Nagel, P., and Pastor, R.: Precise orbit determination for the GRACE mission using only GPS data, J. Geodesy, 80, 322-331, doi:10.1007/s00190006-0073-5, 2006.

Krauss, S., Temmer, M., Veronig, A., Baur, O., and Lammer, H.: Thermospheric and geomagnetic responses to interplanetary coronal mass ejections observed by ACE and GRACE: Statistical results, J. Geophys. Res.-Space, 120, 8848-8860, doi:10.1002/2015JA021702, 2015.

Liu, H., Luehr, H., Henize, V., and Koehler, W.: Global distribution of the thermospheric total mass density derived from CHAMP, J. Geophys. Res., 110, A04301, doi:10.1029/2004JA010741, 2005.

Liu, H., Stolle, C., Forster, M., and Watanabe, S.: Solar activity dependence of the electron density at $400 \mathrm{~km}$ at equatorial and low latitudes observed by CHAMP, J. Geophys. Res., 112, A11311, doi:10.1029/2007JA012616, 2007.

Lu, G., Richmond, A. D., Emery, B. A., and Roble, R. G.: Magnetosphere-ionosphere-thermosphere coupling: Effect of neutral winds on energy transfer and field-aligned current, J. Geophys. Res., 100, 19643-19659, doi:10.1029/95JA00766, 1995.

McNamara, L. F., Cooke, D. L., Valladares, C. E., and Reinisch, B. W.: Comparison of CHAMP and Digisonde plasma frequencies at Jicamarca, Peru, Radio Sci., 42, RS2005, doi:10.1029/2006RS003491, 2007.

Millward, G. H., Moffett, R. J., Quegan, S., and Fuller-Rowell, T. J.: A coupled thermosphere ionosphere plasmasphere model (CTIP), in: STEP Handbook on Ionospheric Models, edited by: Schunk, R. W., Utah State University, 239-279, 1996.

Pawlowski, D. J. and Ridley, A. J.: Quantifying the effect of thermospheric parameterization in a global model, J. Atmos. Sol.-Terr Phy., 71, 2017-2026, doi:10.1016/j.jastp.2009.09.007, 2009. 
Picone, J., Hedin, A., Drob, D., and Aikin, A.: NRLMSISE00 empirical model of the atmosphere: Statistical comparisons and scientific issues, J. Geophys. Res., 107, 1468, doi:10.1029/2002JA009430, 2002.

Rees, M. H.: Physics and chemistry of the upper atmosphere, Cambridge University Press, Cambridge, 1989.

Reigber, C. and Schwintzer, P.: A Challenging Microsatellite Payload for Geophysical Research and Application, in: Small Satellites for Remote Sensing, Space Congress '95, Bremen, 1995.

Richards, P. G. and Voglozin, D.: Reexamination of ionospheric photochemistry, J. Geophys. Res.-Space, 116, A08307, doi:10.1029/2011JA016613, 2011.

Richmond, A. D., Ridley, E. C., and Roble, R. G.: A thermosphere/ionosphere general circulation model with coupled electrodynamics, Geophys. Res. Lett., 19, 601-604, doi:10.1029/92GL00401, 1992.

Ridley, A. J., Deng, Y., and Toth, G.: The Global IonosphereThermosphere Model, J. Atmos. Sol.-Terr. Phy., 68, 839-864, doi:10.1016/j.jastp.2006.01.008, 2006.

Sagalyn, R. C. and Bowhill, S. A.: Progress in Geomagnetic Storm Prediction, in: Geophysical Monograph Series, 01/1993, American Geophysical Union, doi:10.1029/GM073p0157, 1993.

Schunk, R. and Nagy, A.: Ionospheres: Physics, Plasma Physics, and Chemistry, Cambridge Atmospheric and Space Science Series, 2nd Edn., Cambridge University Press, 2009.

Sutton, E. K.: Normalized Force Coefficients for Satellites with Elongated Shapes, J. Spacecraft Rockets, 46, 112-116, doi:10.2514/1.40940, 2009.
Sutton, E. K., Forbes, J. M., and Nerem, R. S.: Global thermospheric neutral density and wind response to the severe 2003 geomagnetic storms from CHAMP accelerometer data, J. Geophys. Res., 110, A09S40, doi:10.1029/2004JA010985, 2005.

Thoemel, J., Singarayar, F., Scholz, T., Masutti, D., Testani, P., Asma, C., einhard, R., and Muylaert, J.: Status of the QB50 cubesat constellation mission, in: 65th International Astronautical Congress, Toronto, Canada, 29 September-3 October 2014, IAC-14.B4.2.11, 2014.

Touboul, P., Foulon, B., and LeClerc, G. M.: STAR, The Accelerometer of the Geodesic Mission CHAMP, in: Proceedings of the 49th IAF Congress, Melbourne, Australia, 28 September2 October 1998, IAF-98-B.3.07, 1998.

Vallado, D. A. and Finkleman, D.: A critical assessment of satellite drag and atmospheric density modeling, Acta Astronaut., 95, 141-165, doi:10.1016/j.actaastro.2013.10.005, 2014.

Zhu, J., Ridley, A. J., and Deng, Y.: Simulating electron and ion temperature in a global ionosphere thermosphere model: Validation and modeling an idealized substorm, J. Atmos. Sol.-Terr. Phy., 138-139, 243-260, doi:10.1016/j.jastp.2016.01.005, 2016.

Zhukov, V. N., Melnikov, E. K., and Smirnov, A. I.: Solving the International Space Station (ISS) motion control long-term planning problem, in: 23rd International Symposium on Space Flight Dynamics, Jet Propulsion Laboratory, 2012. 